\title{
Las políticas de prevención de drogodependencias en las comunidades autónomas españolas: debilidades y fortalezas*
}

\author{
Mercedes Fernández Alonso \\ Universidad de Málaga. Departamento de Derecho del Estado y Sociología \\ Campus de El Ejido, s/n. 29071 Málaga
}

\section{Resumen}

Actualmente, la prevención es la estrategia prioritaria en la lucha contra las drogodependencias en España. No obstante, todavía existen problemas en la planificación y la evaluación de sus políticas, especialmente en el ámbito autonómico. En este trabajo, tras una evaluación diagnóstica basada en técnicas cuantitativas y cualitativas, se reflejan los principales rasgos y carencias de las políticas de prevención en las comunidades autónomas. Con ello, se pretende realizar una aportación al estudio y a la investigación de esta línea de actuación.

Palabras clave: bienestar social, política social, drogas, autonomías.

\begin{abstract}
Drug prevention policies in the autonomous regions in Spain: shortcomings and strengths
\end{abstract}

Nowadays, drug prevention is the main performance objective of drugs policies in Spain. Nevertheless, there are still notable shortcomings in planning and evaluation of these policies, especially concerning the Autonomous Regions. In this paper, across a diagnostic evaluation based on a combination of quantitative and qualitative techniques, the principal features and shortcomings about autonomic prevention policies are provided. The obtained results are considered of great utility.

Key words: social welfare, social policy, drugs, autonomous regions.

* En este trabajo se ofrecen algunos de los resultados obtenidos en una investigación más amplia sobre el Plan Nacional sobre Drogas y los planes autonómicos (proyecto subvencionado por la Delegación del Gobierno para el PNSD, Ministerio del Interior), que fue presentada como tesis doctoral en la Universidad de Granada en 2004, bajo la dirección del profesor Iglesias de Ussel y del profesor Trinidad (codirector). La autora agradece los comentarios y sugerencias de los miembros del tribunal, de los profesores Gobernado y Jaime y, de manera especial, de Gregor Burkhart, especialista en prevención en el Observatorio Euopeo de la Droga y las Toxicomanias (OEDT). 


\section{Sumario}

Introducción

1. Planteamiento del problema

2. Características metodológicas del estudio
3. Resultados de la investigación.

Las políticas de prevención en las comunidades autónomas

4. Aportaciones finales. Conclusiones Referencias bibliográficas

\section{Introducción}

El artículo ofrece un análisis de las políticas de prevención de drogas en el ámbito autonómico en España. A su vez, al ser medidas en base a los mismos parámetros, muestra una visión comparativa entre las diferentes regiones. En general, se trata de una evaluación diagnóstica que busca conocer el problema, las necesidades y sus características básicas para hacerle frente. Para ello, se ha podido contar con el punto de vista de los responsables autonómicos, aspecto novedoso en las investigaciones sobre esta materia realizadas en España. En definitiva, un estudio de carácter explicativo que investiga el porqué de los logros y de los fracasos, al objeto de conocer las fortalezas y las debilidades y proponer acciones de mejora.

Desde el punto de vista de la sociología, la mayor parte de los autores (véase Recio, 1997) contempla el fenómeno de la droga como «capital simbólico y hecho social total», es decir, éste cumple una serie de funciones y está implicado en la estructura social global. Tomando como referencia a sociólogos como Bourdieu y Giddens, la pluralidad y el alto grado de diferenciación estructural de la sociedad postmoderna ha llevado a la propagación de universos simbólicos parciales, y uno de ellos es el que se ha formado en torno a la droga.

Las políticas públicas aplicadas al contexto de las drogodependencias han sido objeto de gran interés y han suscitado un conjunto de respuestas desde mediados de los años setenta en nuestro país. Sin embargo, es a partir de 1985, con la aprobación, por parte del Consejo de Ministros, del Plan Nacional sobre Drogas (PNSD), cuando se establece una política verdaderamente coordinada en esta materia. El Plan es entendido como «una iniciativa gubernamental destinada a coordinar y potenciar las políticas que, en materia de drogas, se lleven a cabo desde las distintas administraciones públicas y entidades sociales en España».

Esta política ha ido renovándose y adaptándose a las nuevas necesidades. En 1997, destaca, como eslabón intermedio, el Plan de Medidas para luchar contra las Drogas y, más recientemente, en 1999, la aprobación de la Estrategia Nacional sobre Drogas 2000-2008. Con el fin de avanzar en el logro de los objetivos propuestos y tras la evaluación del primer tramo de vigencia de la Estrategia ${ }^{1}$, surge el actual Plan de Acción sobre Drogas 2005-2008².

1. Con la intención de mejorar las actuaciones en materia de drogas que se emprendiesen, se establecieron los años 2003 y 2008 como fechas de referencia para su evaluación.

2. Dicho plan está en sintonía con la Estrategia Europea sobre Drogas 2005-2012. En <www.pnsd.msc.es>. 
El Plan Nacional surgió para dar respuesta a la gran alarma social despertada en la década de 1980 entre los españoles en torno al problema de la heroína y las consecuencias que se derivaban de él, especialmente sanitarias. Sin embargo, pese a la importancia del perfil asistencial dominante en esos momentos ( $80 \%$ de los recursos destinados a la asistencia sanitaria), la prevención siempre ha estado presente ${ }^{3}$. La relevancia de la estrategia preventiva comienza a tener calado cuando, con el paso de los años, la red asistencial se encuentra notablemente asentada y se van produciendo cambios, tanto en el consumo de drogas como en las necesidades asociadas al mismo. Así, en el Plan de Medidas para luchar contra las Drogas, aprobado por el Consejo de Ministros el 24 de enero de 1997, se expresa que el Gobierno adopta la prevención como «la máxima prioridad entre sus actuaciones». Más adelante, con la ratificación de la Estrategia Nacional, su primacía se sigue manteniendo, aunque con un talante diferente y adaptado a las nuevas circunstancias.

En resumen, a la Delegación del Gobierno para el PNSD, como máximo órgano responsable de la ejecución del Plan, le compete definir una política estratégica global que, desde el consenso, posibilite la estabilidad de los programas y las actuaciones preventivas, su constante evaluación y su progresiva mejora. Las comunidades autónomas, en colaboración con las administraciones locales y el movimiento asociativo, tienen la función de planificar y ejecutar unas adecuadas políticas autonómicas y locales en esta temática, mediante sus correspondientes apoyos técnicos y financieros.

A pesar del notable interés en las políticas de prevención en todos los niveles de la Administración y en los diferentes ámbitos sociales, en general, existe un desconocimiento sobre los efectos reales que tienen sus programas y sus actuaciones. En nuestro país, habría que destacar la falta de estudios comprehensivos en este sentido, especialmente si se tiene en cuenta el interés social que suscita, por ejemplo, si se considera la cantidad de recursos presupuestarios que se invierten.

Desde el punto de vista del conocimiento sociológico, el análisis de este fenómeno es fundamental. Los cambios que se han producido en la sociedad han afectado de igual manera al consumo de sustancias y a sus patrones de uso, hasta el punto que ha pasado a ser considerado uno de los problemas sociales más acuciantes en la actualidad. Las drogas se han convertido en una parte más de la vida cotidiana, se ha producido un proceso de normalización de su uso, fundamentalmente entre los jóvenes (Mejías, 2000). En esta línea, conocer los efectos reales de la prevención se ha establecido como una tarea esencial de esta investigación, al ser considerada la estrategia más eficaz para enfrentarse a este fenómeno social.

El momento escogido para la realización de la investigación es el primer periodo de vigencia de la Estrategia Nacional (de los años 2000 al 2002), inter-

3. En la presentación del documento base del plan, el Ministro de Sanidad y Consumo manifestaba la idoneidad de las acciones preventivas como el punto de partida ante cualquier actuación en materia de drogodependencias. 
valo de tiempo especialmente relevante, al establecerse con su creación (en 1999) el año 2003 como la fecha de referencia para proceder a una primera evaluación parcial de ámbito nacional (la segunda y total no será hasta el 2008). Este estudio complementa la evaluación de la situación de España en materia de drogodependencias en un momento que fue considerado clave, de cara al futuro desarrollo de las políticas sobre drogas.

La estructura del artículo se divide en cuatro secciones. En primer lugar, el planteamiento del problema, donde se especifica qué nos ha llevado al estudio de esta situación y se realiza un primer acercamiento a la materia. A continuación, las características metodológicas del estudio: el método, las técnicas, los indicadores de evaluación y la descripción del modelo empleado. El tercer apartado se centra en los resultados de la investigación, concretamente, las políticas de prevención en las comunidades autónomas: rasgos generales, planificación y, finalmente, las fortalezas, las debilidades y los retos. El artículo se cierra con un apartado de conclusiones.

\section{Planteamiento del problema}

A pesar de que la prevención es considerada la estrategia prioritaria y la más adecuada para enfrentarse a la problemática de la drogadicción, llama la atención que se sigan registrando en la actualidad, especialmente en el ámbito autonómico, debilidades y carencias en algunos de sus componentes. Tras una revisión de diferentes trabajos de especialistas en la materia en nuestro país (véase, entre otros, Becoña, 1999, o Acero, 1997), se aprecia una falta de recursos, una escasa continuidad de las actuaciones, dificultades metodológicas (planificación y evaluación), problemas de coordinación, leve implicación del personal, carencia de sistemas de registro de las actuaciones y necesidad de mayor atención a los colectivos de riesgo y al trabajo con las familias, entre otros elementos.

En el ámbito autonómico se han encontrado dificultades especiales. El problema principal, de acuerdo con el PNSD, es que la información proporcionada por las comunidades es insuficiente, y resulta, en muchas ocasiones, difícil conocer la situación real de las mismas. Por ejemplo, todas realizan programas de prevención con variados objetivos, poblaciones, metodologías, etc., por lo que la forma de registro de las mismas y la presentación de datos concretos es muy difícil. En segundo lugar, las fichas de recogida de información sobre la labor desarrollada con indicadores de la actividad en los diferentes ámbitos, hace que la mayor parte de la información que se recoge sea de tipo cuantitativo, lo que dificulta una adecuada interpretación y comprensión de la realidad social de cada territorio. Como un último aspecto, también se hace escasa referencia a temas de investigación y evaluación.

La existencia de estos problemas a la hora de conocer con profundidad las actuaciones de las comunidades, hace casi imposible la elaboración de un análisis significativo a escala nacional. Esto pone en evidencia que la evaluación de la prevención debe ser una realidad presente a la hora de valorar si las actua- 
ciones emprendidas son las adecuadas y si están produciendo los efectos deseados ${ }^{4}$.

\section{Características metodológicas del estudio}

La prevención, ante la dificultad de constatar sus efectos, continuamente se enfrenta a críticas y a la necesidad incesante de demostrar la calidad y la eficiencia de sus actividades. Con la celebración de la Primera Conferencia Europea sobre Evaluación de Prevención de Drogas, por el Observatorio Europeo de las Drogas y las Toxicomanías (OEDT) en marzo de 1997, se llegó a la conclusión de que esta práctica ha de tener un papel central en las actividades preventivas. Resulta imprescindible demostrar que éstas se basan en modelos teóricos y en estrategias de acción predefinidas, que siguen unos objetivos establecidos y operacionales, que se adentran en el campo de la multidisciplinariedad y la flexibilidad y que ofrecen la posibilidad de una metodología de control de calidad accesible a los profesionales de los distintos países (Burkhart, 1998) $)^{5}$

Siguiendo la línea europea, en España también se ha impulsado la evaluación en el campo de las drogodependencias. En el área de prevención, el Plan Nacional ha favorecido el desarrollo de programas globales, con metodologías rigurosas y susceptibles de evaluación. En 1996, en la sesión de la Comisión Técnica Interautonómica, resultó ser un acuerdo unánime la creación de una ponencia técnica para establecer los criterios primordiales de homologación que debían seguir estas acciones en todo el Estado, sometidas a revisión con el transcurso de los años. Fruto de este trabajo fue el documento Criterios básicos de intervención de los programas de prevención de drogodependencias (PNSD, 1996).

Pese a los avances conseguidos, todavía queda una asignatura pendiente: resulta difícil precisar el número exacto de actividades y programas preventivos existentes y, sobre todo, cuántos son evaluados ${ }^{6}$. Son necesarios mayores progresos científicos, recursos, el desarrollo de investigaciones sistemáticas y que la evaluación se convierta en una parte integrante o normalizada de la intervención (véase Salvador, 2001; Kröger, Winter y Shaw, 1998; o Leukefeld y Bukoski, 1997). Según Francisco Alvira (2000), una toma de decisiones basa-

4. Frente a esta situación, la Estrategia Nacional sobre Drogas (1999: 46-53) plantea una serie de objetivos generales sobre prevención, con los correspondientes indicadores («de nueva aplicación») que se han considerado más idóneos para la comprobación de la consecución de los mismos en los momentos marcados para su evaluación: los años 2003 y 2008.

5. El Manual para la Evaluación de la Prevención, probado y mejorado sobre la base de programas de diferentes países de la Unión, es el resultado de este esfuerzo colectivo entre un gran número de nacionalidades.

6. A pesar de que se deba continuar trabajando en este campo, cada vez se está avanzando más en el diseño y en la construcción de un sistema de instrumentos perfectamente validados y contrastados que constituyan la base para ayudar y guiar el proceso de evaluación de las actividades preventivas: el cuestionario Idea-Prevención/EDDRA (PNSD, 2000). 
da a la vez en la razón y en la realidad empírica permite mejorar la calidad de nuestras intervenciones, por lo que la evaluación es garantía de calidad de las mismas.

En esta investigación, se ha desarrollado un modelo de evaluación diagnóstica. En general, se trata de conocer el problema o las necesidades y sus características básicas. Se ha planteado una evaluación constructiva de carácter externo y global, con el objeto de indagar en las causas o en los factores relacionados con los logros y los fracasos para proponer líneas de mejora. Se ofrece una visión integral (no sólo de los programas y las intervenciones) de la prevención en España, en general, y en las comunidades autónomas, en particular, mediante una perspectiva comparada entre las diferentes regiones.

El estudio se ha realizado a través de dos tipos de fuentes: las secundarias y las primarias. En lo relativo a las primeras, se ha utilizado como técnica de investigación el análisis documental (revisión bibliográfica y búsqueda de datos estadísticos). En un principio, se ha tratado de examinar toda la información relativa al Plan Nacional, a los planes autonómicos y, en general, al ámbito de las drogodependencias, como guía para la posterior construcción de los instrumentos de recogida de información. Estos datos han sido completados con los procedentes de otras instituciones, entre ellas, el Centro de Investigaciones Sociológicas, el Instituto Nacional de Estadística o los Ministerios del Interior, de Sanidad y Consumo, etc.

En lo referente a las fuentes primarias, se han empleado dos técnicas para la recopilación de datos: el cuestionario y la entrevista semiestructurada, ésta última ha permitido el enriquecimiento y el contraste de la información (oportunidad de clarificar ideas en un contexto de interacción directo). Se ha llevado a cabo una combinación de procedimientos cuantitativos y cualitativos que ponen en evidencia la importancia que ha tenido a lo largo de este estudio la triangulación metodológica. En opinión de Udo Kelle (2001), se trata de la «integración de diferentes perspectivas en los fenómenos investigados», una investigación sociológica que combina lo cualitativo y lo cuantitativo para responder a los interrogantes que surjan y «ayudar en la realización de una mirada más profunda sobre los aspectos teoréticos de la integración metodológica en sociología».

Para la evaluación, se han considerado tres dimensiones: los rasgos generales de la prevención en los planes autonómicos, la planificación de las intervenciones y los principales resultados de sus políticas. Los indicadores a evaluar en cada uno de éstos han hecho referencia, en lo que al primero se refiere, a los aspectos más generales de esta área (objetivos, destinatarios, ámbitos de actuación, difusión, estudios e investigaciones). En segundo lugar, en la planificación, se han basado en las subdimensiones que caracterizan el desarrollo de la planificación y la coordinación con las distintas administraciones públicas, con la iniciativa social y con otras entidades. Finalmente, los indicadores a evaluar en el bloque de resultados han tratado de medir los principales logros, efectos o consecuencias derivadas de las actuaciones emprendidas (ver la tabla I). 
Tabla I. Dimensiones e indicadores sobre prevención a evaluar

\begin{tabular}{|c|c|}
\hline Dimensiones & Indicadores a evaluar \\
\hline Rasgos generales de la prevención & $\begin{array}{l}\text { - Prioridad de esta área de intervención. } \\
\text { - Objetivos. } \\
\text { - Destinatarios. } \\
\text { - Ámbitos de actuación. } \\
\text { - Difusión a la población de las labores, } \\
\text { los programas o las actividades. } \\
\text { - Estudios e investigaciones que se } \\
\text { realizan. }\end{array}$ \\
\hline $\begin{array}{l}\text { Planificación de las intervenciones } \\
\text { preventivas }\end{array}$ & $\begin{array}{l}\text { - Encargados de la prevención } \\
\text { en las comunidades autónomas } \\
\text { (Organigrama de la Oficina de } \\
\text { Coordinación de los Planes } \\
\text { Autonómicos). } \\
\text { - Realización de estudios previos de } \\
\text { necesidades y fuentes empleadas. } \\
\text { - Elaboración de mapas de necesidades } \\
\text { y definición de poblaciones con alto } \\
\text { riesgo. } \\
\text { - Recursos: } \\
\text { a) Humanos (número, perfil del } \\
\text { personal, evolución, principales } \\
\text { problemas). } \\
\text { b) Económicos (presupuesto, } \\
\text { evolución, distribución por áreas). } \\
\text { c) Materiales (tipología). } \\
\text { - Coordinación (implicación y } \\
\text { participación de otros sectores, } \\
\text { ONG). }\end{array}$ \\
\hline Resultados de las políticas preventivas & $\begin{array}{l}\text { - Aspectos positivos o logros alcanzados } \\
\text { - Problemas o puntos débiles. } \\
\text { - Retos. }\end{array}$ \\
\hline
\end{tabular}

Fuente: elaboración propia.

Como sujetos informadores, se han seleccionado a los responsables de los planes autonómicos ${ }^{7}$. Los motivos estriban en el hecho de haber sido definido un tipo de evaluación basada en el conocimiento global de la situación, considerándose, por lo tanto, a estos colectivos como los más idóneos. A su vez, sus puntos de vista se consideran innovadores, puesto que no existen, en el ámbi-

7. Aunque, de cara al conocimiento detallado de las diferentes actuaciones y en los casos en los que ha sido necesario, se ha contemplado también la opinión de técnicos, jefes de gabinete o coordinadores. 
Planes autonómicos

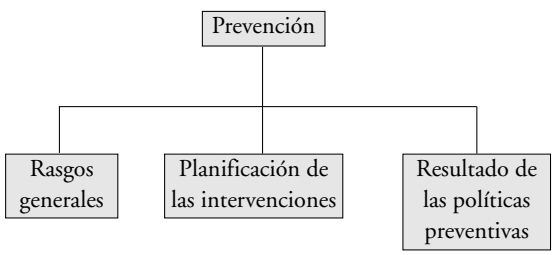

Triangulación de métodos

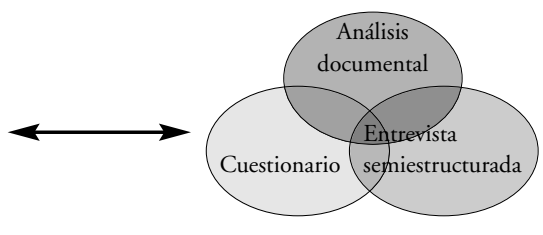

Fase I: Descriptivo

Procedimiento a seguir 1. Análisis de fuentes secundarias.

2. Envío a los responsables autonómicos de los cuestionarios de recogida de información

3. Elaboración de los primeros informes de cada comunidad.

4. Análisis de la información del cuestionario.

Fase II: Validación y contrastación de la fase descriptiva

Procedimiento a seguir 1. Trabajo de campo: entrevistas semiestructuradas a responsables autonómicos.

2. Análisis y triangulación de resultados.

Fase III: Informe preliminar

Procedimiento a seguir 1. Elaboración de informes de resultados de cada comunidad.

2. Envío de informes a responsables PNSD y planes autonómicos para la emisión de un juicio de valor.

Fase IV: Informe final

Gráfico I. Descripción del modelo de evaluación diagnóstica. Fuente: elaboración propia.

to nacional, estudios e investigaciones centrados en el análisis de sus opiniones y maneras de valorar las políticas autonómicas sobre drogas.

\subsection{Descripción del modelo}

El modelo de evaluación diagnóstica, debido a la envergadura del estudio y a las necesidades que han ido surgiendo en el transcurso del mismo, se ha desarrollado en cuatro fases. En primer lugar, la descriptiva, basada en el análisis de las diferentes acciones llevadas a cabo en los planes autonómicos, especialmente en el área de prevención, a través de la explotación de datos secundarios y de la opinión de los responsables de cada comunidad (cuestionario).

En segundo lugar, una fase de validación y contrastación de la etapa anterior, por medio de entrevistas semiestructuradas y de la triangulación de métodos. A continuación, la realización de un informe preliminar con los principales resultados obtenidos — gracias al empleo de los diferentes métodos y técnicas de investigación - para ser enviado, en un primer momento, a los 
responsables del PNSD y, seguidamente, de los planes autonómicos, para la emisión de un juicio de valor. Para finalizar, el estudio se ha cerrado con la elaboración, por parte del investigador, de un informe final o de conclusiones, en el que se exponen los rasgos más característicos de la prevención, la realidad de cada territorio autonómico, las principales debilidades, fortalezas y retos (ver gráfico I).

\section{Resultados de la investigación. Las políticas de prevención en las comunidades autónomas ${ }^{8}$}

El Plan Nacional sobre Drogas encuentra su plasmación territorial a través de los planes autonómicos vigentes en las comunidades que conforman el Estado español. Su puesta en marcha ha permitido disponer de unas estructuras de intervención y desarrollar redes asistenciales y programas preventivos (Estrategia Nacional sobre Drogas, 1999). Un plan constituye una iniciativa política que define el marco de futuras actuaciones, un conjunto de medidas de coordinación, planificación y acción, para un periodo de tiempo determinado y sometido a un posterior sistema de evaluación (véase Arenilla, 2000).

Los planes autonómicos contemplan distintas áreas, una de ellas la prevención. Departamentos o secciones específicas, con el apoyo de grupos de trabajo técnico en colaboración con otros sectores (educativo, sanitario, etc.), se encargan de su desarrollo. Con ello, se ponen en marcha - con los correspondientes recursos - intervenciones y programas informativos y formativos en los diferentes ámbitos relacionados: escolar, comunitario, familiar, laboral, medios de comunicación y otros.

8. La mayor parte de la información que se presenta es aquélla que se ha obtenido en el estudio realizado, principalmente a través de los cuestionarios y las entrevistas, por lo que, generalmente, no se han podido incluir a las autonomías que no han participado en el mismo (Baleares y Canarias, en un principio, y Madrid, posteriormente) o las que, aún habiendo colaborado, no han proporcionado datos al respecto. No obstante, este inconveniente ha tratado de ser solventado mediante el análisis de fuentes secundarias, aunque no siempre se ha obtenido el éxito esperado.

A la hora de interpretar los resultados, hay que tener en cuenta, por ejemplo, que algunas comunidades denotan una gran trayectoria en esta materia, una sólida base sobre la que asentar sus actuaciones. Esta situación se encuentra en relación con factores como la antigüedad en la ejecución de políticas coordinadas en drogodependencias (ciertamente pioneras Andalucía, Asturias, Cantabria, las dos Castillas, Galicia, Madrid y La Rioja) o el número de planes autonómicos puestos en práctica. Asimismo, debería ser considerado el momento en el que se elaboran las primeras leyes sobre drogas (Castilla y León, el País Vasco y Cataluña). Aunque esta situación no sea determinante a la hora de comparar las comunidades, sí que se aprecia al analizar determinados aspectos de la planificación, la coordinación y la evaluación de las intervenciones.

En lo referente a los datos que aparecen, son los que han sido proporcionados. No obstante, a raíz de este estudio, se abre una puerta a futuras investigaciones, con el fin de contrastar esta información de la mano de otros colectivos implicados. 


\subsection{Rasgos generales de la prevención}

La prevención, al igual que en el ámbito nacional, ha pasado a ser el objetivo principal para la mayoría de las comunidades consultadas. En concreto, en nueve de ellas (Aragón, Baleares, Cantabria, Castilla-La Mancha, Ceuta, La Rioja, Melilla, Navarra y el País Vasco) es considerada la línea central de actuación, y en otras como Andalucía, Asturias, Castilla y León y Extremadura, aunque compartiendo el protagonismo con terceras (incorporación social, reducción de daños o intervención asistencial), también es señalada como prioritaria. No obstante, hay otras cuyas actuaciones primordiales van encaminadas en otro sentido, véase el caso de Galicia (a la inserción social) o Valencia (a la intervención asistencial) (ver tabla II). Se observa que todavía en determinadas comunidades no se concibe como el eje central, puesto que sus políticas están enfocadas en otro sentido.

Los objetivos (PNSD, 1996-2003) de la prevención están encaminados, fundamentalmente, hacia dos líneas. En primera instancia, en un nivel más básico?: impedir el consumo de drogas, dar a conocer los problemas que esán relacionados con él, controlar la oferta y la demanda, informar, formar, educar y modificar actitudes y comportamientos. Seguidamente, en un grado más profun$\mathrm{do}^{10}$ : evitar que el consumo habitual se convierta en drogodependencia; mejorar la calidad de vida de los afectados por este problema, incidiendo en los factores que lo suscitan; prevenir el abuso y la dependencia, y reducir los daños relacionados con las drogas. En definitiva, disminuir y acabar con los factores asociados con el consumo, a través de un conjunto de programas, actividades y recursos coordinados y evaluados. Estos propósitos, plasmados en intervenciones específicas, se dirigen a diversos sectores de la población, tal es el caso de los niños, los adolescentes y los jóvenes; las familias; la comunidad escolar; los trabajadores; las personas no iniciadas en el consumo; los drogodependientes y diversas entidades u organismos, en una palabra: la comunidad. Estas actuaciones se agrupan en una serie de ámbitos, habitualmente el comunitario, el educativo o escolar, el familiar, el laboral y el de los medios de comunicación.

Otro aspecto es la difusión a la población de las labores, programas o actividades que desarrolla el plan y el perfil de los sujetos a la que va destinada. Todas las comunidades afirman desarrollar esta tarea, los programas de prevención se encuentran dentro del grupo de los que se suministra mayor información, con la excepción de Extremadura (en mayor medida, centrada en los asistenciales) y el País Vasco (no existe ningún tipo prioritario). El principal destinatario de la información es el conjunto de la población, aunque también se indican colectivos concretos como las familias, los adolescentes y los jóvenes,

9. Coincidiendo con los objetivos que se persiguen en la prevención primaria: trabajar entre personas que no han experimentado ningún contacto con el mundo de las drogas.

10. Se haría referencia al trabajo con personas que ya han probado las drogas, bien cuando ya se ha detectado alguna situación relacionada con el consumo (prevención secundaria), bien cuando se avista un uso problemático (prevención terciaria). 
Tabla II. Áreas prioritarias de intervención en los planes autonómicos sobre drogas

\begin{tabular}{ll}
\hline Comunidades & Áreas prioritarias de intervención \\
\hline Andalucía & Prevención, incorporación social y reducción de daños. \\
Aragón & Prevención (modelo de «intervención preventiva») (1). \\
Asturias & Prevención, asistencia y reinserción (2). \\
Baleares & Prevención. \\
Cantabria & Prevención. \\
Castilla-La Mancha & Prevención. \\
Castilla y León & Intervenciones en materia de alcohol y tabaco (control de la \\
& oferta e investigación), prevención, inserción sociolaboral. \\
Ceuta & Prevención. \\
Extremadura & Prevención y reincorporación sociolaboral. \\
Galicia & Inserción social. \\
Melilla & Prevención. \\
Navarra & Prevención. \\
País Vasco & Prevención. \\
La Rioja & Prevención. \\
Valencia & Intervención asistencial. \\
\hline
\end{tabular}

(1) Modelo integral en el que se confunde asistencia y prevención.

(2) Siempre se ha dado mayor importancia a la asistencia, pero ahora están más centrados en la prevención.

Fuente: elaboración propia a partir de los datos suministrados en las entrevistas a los responsables y en las memorias del PNSD y de los planes autonómicos.

los mediadores sociales, los técnicos municipales, los trabajadores, los empresarios o la comunidad escolar.

En materia de estudios e investigaciones, se ha hecho especial incidencia en los estimados deficitarios. En primera instancia, los relacionados con los factores de riesgo y protección del consumo de drogas y, en segunda instancia, los relativos a la evaluación — de implementación y de resultados - de los programas preventivos. De las trece comunidades que han suministrado información, cinco de ellas (las dos Castillas, Extremadura, Navarra y La Rioja) confirman no realizar estudios ni investigaciones sobre los mencionados factores. Aquí conviene resaltar la importancia de su análisis, ya que, en resumen, la prevención se centra en evitar los factores de riesgo y desarrollar los de protección. Esto es, impedir las «condiciones que facilitan el consumo o que aumentan la posibilidad de que surja este problema» y fomentar las que, por el contrario, "contribuyen a disminuir el riesgo de que una persona consuma drogas", es decir , las que "hacen a uno menos vulnerable al consumo, facilitando el rechazo a las drogas» (Ríos, Espina y Baratas, 1997).

En cuanto a los estudios de evaluación de programas, dos regiones (CastillaLa Mancha y Murcia) no contemplan la investigación en la modalidad de la 
implementación, cifra que se duplica (Cantabria, Murcia, País Vasco y La Rioja) en la de resultados. En opinión de los responsables, hay que seguir profundizando en estas materias y, en el ámbito nacional, habría que aumentar los estudios sobre jóvenes, familias y colectivos de riesgo; espacios de ocio; motivaciones del consumo y, en definitiva, la investigación general de la prevención.

\subsection{Planificación}

Como punto de partida, atendiendo al organigrama de la Oficina de Coordinación de los planes, los encargados de la prevención son departamentos, áreas, secciones o servicios específicos, contando con grupos de trabajo técnico, coordinadores o responsables y dependiendo, generalmente, de la Dirección General o de una secretaría técnica de drogodependencias. En otras autonomías, como en Ceuta, está el Instituto para la Prevención de las Drogodependencias; en el País Vasco, el Centro Coordinador de Drogodependencias (DAK), y la Oficina de Atención al Ciudadano, en La Rioja.

En la planificación de las intervenciones, realizar un análisis previo de la situación, en base a estudios, investigaciones, sistemas de información o encuestas en el propio ámbito regional o local, es una pauta compartida. Sin embargo, como complementario marco de referencia, en parte del territorio (Cantabria, Castilla-La Mancha, Cataluña o La Rioja), también se utilizan las fuentes de ámbito nacional.

En este proceso, se elabora un mapa de necesidades (con la excepción de Cantabria, Castilla y León, Ceuta, Extremadura y Navarra), con el fin de detectar los puntos prioritarios de actuación. Así, se definen poblaciones de intervención prioritaria o de alto riesgo — generalmente población juvenil, alumnos con dificultades especiales o comportamiento antisocial, hijos de drogodependientes o familias y menores marginados- casi en la totalidad de las comunidades (menos en Cantabria, Ceuta, Extremadura y el País Vasco) (ver tabla III).

Para conseguir los citados objetivos y poner en práctica las actuaciones, en cualquier intervención son necesarios recursos. En lo que se refiere a los humanos, se avistan especiales dificultades. Por ejemplo, en cuanto al número, hay grandes contrastes: algunas regiones cuentan con altas dotaciones de personal para el desempeño de las tareas preventivas (Galicia y Aragón), frente a otras con apenas profesionales dedicados a esta labor (Cantabria o Melilla). Profundizando en el perfil del personal, son principalmente licenciados (psicólogos y pedagogos), en su totalidad, en Cantabria y en Extremadura y, mayoritariamente, en Galicia, Valencia, Castilla-La Mancha y Murcia. A continuación, sobresalen los diplomados, básicamente trabajadores sociales, en Asturias, Aragón y Ceuta. Junto con ellos, también desarrollan tareas personas con estudios de bachiller y de formación profesional (Aragón, Castilla-La Mancha, Ceuta, Galicia, Murcia y Valencia) o, en algunos casos, con el graduado escolar (Ceuta y La Rioja) (ver tabla IV).

Siendo la prevención el objetivo principal de gran parte de los planes, aunque en la mayoría haya aumentado en los últimos años, todavía se considera defi- 
Tabla III. Elaboración de mapas de necesidades y definición de poblaciones de alto riesgo

Definición de poblaciones de intervención prioritaria Comunidades Mapa de necesidades o de alto riesgo

Andalucía

Sí (población juvenil y general de zonas de alto riesgo social).

\begin{tabular}{lll}
\hline Aragón & Sí & SÍ (jóvenes y la reducción del daño). \\
\hline Asturias & & Sí (alumnos de la ESO). \\
\hline Cantabria & NO & NO. \\
\hline
\end{tabular}

Castilla y León NO

SÍ (estudiantes con fracaso escolar e incidencia en un bajo compromiso con la escuela; niños, adolescentes y jóvenes con comportamiento antisocial; hijos de alcohólicos y toxicómanos, etc.).

Cataluña SÍ

Sí (determinadas a partir de estudios epidemiológicos clave. Entre otras, la población ubicada en zonas de atención preferente, jóvenes usuarios de los servicios de ocio nocturno durante los fines de semana y los hijos de drogodependientes).

\begin{tabular}{|c|c|c|}
\hline Ceuta & NO & NO \\
\hline Extremadura & NO & NO \\
\hline Galicia & $\begin{array}{l}\text { Sí (mapa de riesgo } \\
\text { epidemiológico) }\end{array}$ & $\begin{array}{l}\text { SÍ (menores institucionalizados, hijos de } \\
\text { drogodependientes y adolescentes y jóvenes } \\
\text { con problemática escolar). }\end{array}$ \\
\hline Madrid (1) & Mapa de riesgos & \\
\hline Melilla & & SÍ \\
\hline Navarra & $\mathrm{NO}$ & SÍ (cada municipio las define en su propio entorno). \\
\hline País Vasco & Sí & NO (2). \\
\hline La Rioja & Sí & Sí (familias y menores marginados). \\
\hline Valencia & Sí & $\begin{array}{l}\text { SÍ (ámbito escolar: niños de doce años en adelante; } \\
\text { ámbito comunitario: los hijos de padres } \\
\text { alcohólicos, como experiencia piloto; ámbito de la } \\
\text { atención «reducción de daño": un programa de } \\
\text { prevención terciaria para población de alto riesgo). }\end{array}$ \\
\hline
\end{tabular}

(1) En PNSD. Memoria 2000, p. 200.

(2) Aunque no se definen, hay planes de prevención dirigidos a distintas poblaciones desde estructuras cercanas a ellas (por ejemplo, gitanos).

Fuente: elaboración propia a partir de los datos suministrados en el cuestionario de recogida de información a los responsables autonómicos. 
citario el volumen de personal. A su vez, los responsables hacen referencia a otros problemas, como la inestabilidad laboral, las dificultades de implicación y colaboración de algunos profesionales y entidades, la necesidad de mayores infraestructuras y la falta de especialización y de formación. Tras el análisis, se aprecia que hablamos de un campo en continuo cambio y en el que el reciclaje de los trabajadores es clave para poder llegar realmente y de la manera más adecuada al conjunto de la población. Una solución práctica sería la creación de un plan o de una unidad específicamente encargada de esta tarea ${ }^{11}$.

En cuanto a los recursos económicos, es un rasgo positivo el aumento del presupuesto destinado a la prevención con el paso de los años (de 3.122.000 euros en 1986 a 43.505 .000 en el 2003), aunque no ocupa todavía el lugar que le corresponde en la partida de gastos de las comunidades. Es lógico encontrar los mayores niveles registrados en las áreas de asistencia y reinserción, dado el elevado número de personal y el coste de los servicios y de los recursos desplegados para la atención y el tratamiento de drogodependientes. Sin embargo, si la prevención ha pasado a ser el área prioritaria, no es razonable que todavía existan diferencias tan marcadas en el conjunto del territorio entre el gasto destinado a ambas áreas de actuación (un 18,7\% a prevención y un 73,71\% a asistencia y reinserción, del total del presupuesto, en el año 2003) (PNSD, 2003: 40).

Por comunidades (ver gráfico II), las que mayor presupuesto han destinado a la prevención en los últimos años, en relación con el total del que se dispone, han sido Castilla-La Mancha (36,5\%), La Rioja (36,2\%), Aragón $(33,4 \%)$, Melilla $(31,1 \%)$ y Cantabria $(28,1 \%)$. Esto se encuentra en concordancia con el hecho de que en ellas se considere esta área como la principal línea de actuación. Caso contrario se aprecia en Baleares (25,2\%), País Vasco $(19,2 \%)$, Navarra $(13,7 \%)$ y Ceuta $(12,6 \%)$, donde, aún afirmando la prioridad de la prevención, le designan un presupuesto medio, en el caso de las dos primeras, o se encuentra entre las que menos dinero le destinan, en el caso de las dos últimas ${ }^{12}$. Para finalizar, mencionar a Castilla y León, donde, a pesar de que sus primacías también sean las intervenciones en materia de alcohol y tabaco (control de la oferta e investigación) y la inserción sociolaboral, se posiciona entre las de menor gasto, acompañada por Murcia y Cataluña.

En la coordinación, necesaria para la puesta en marcha de las intervenciones, se observa una disparidad en la manera de relacionarse del plan con otros sectores (sanitario, asuntos sociales, educación, política, etc.). Aunque lo más extendido sean las comisiones y los convenios, algunas citan también subven-

11. Como ya se tiene, por ejemplo, el Servicio de Salud, en Asturias, u otros más generales, como el de Prevención, Reinserción y Coordinación, en Galicia; el Aula de Formación para la Prevención, en Canarias; Planes de Formación, en Cantabria y Ceuta; la Escuela de Administración Pública y la de Salud, en Extremadura; la Unidad de Educación para la Salud, en Murcia; la Dirección Técnica del Plan, en Navarra, y el Instituto de Investigación en Drogodependencias (INID), en Valencia.

12. Téngase en cuenta, para el caso del País Vasco, la referencia situada al pie del gráfico II. 
Tabla IV. Rasgos generales del personal que trabaja en temas de prevención (excluida la Oficina de Coordinación del Plan)

\begin{tabular}{|c|c|c|c|c|}
\hline Comunidades & Cantidad & Perfil mayoritario & Evolución & Problemas \\
\hline Andalucía & Suficiente. & Personal técnico y agentes de prevención. & & \\
\hline Aragón & Insuficiente. & $\begin{array}{l}\text { Diplomados (77), licenciados (36), } \\
\text { bachiller y FP (11). }\end{array}$ & Aumento. & \\
\hline Asturias & Insuficiente. & Diplomados (trabajadores sociales). & Constante. & \\
\hline Cantabria & Suficiente. & Licenciados. & Constante. & \\
\hline Castilla-La Mancha & Suficiente. & $\begin{array}{l}\text { Licenciados (37), diplomados (8), } \\
\text { bachiller y FP (8). }\end{array}$ & Aumento. & Eventualidad de los contratos. \\
\hline Castilla y León & Insuficiente. & & Aumento. & $\begin{array}{l}\text { Falta de formación, de especialización y de dotación de estrategias más eficaces } \\
\text { (protocolos, preguntas, etc.). Necesidad de aumentar el personal, especialmente } \\
\text { en tareas de supervisión, apoyo y seguimiento técnico mediad. prev. }\end{array}$ \\
\hline Cataluña & & $\begin{array}{l}\text { Técnicos en prevención, colaboradores } \\
\text { en formación y prev. y administrativos. }\end{array}$ & & \\
\hline Ceuta & Insuficiente. & $\begin{array}{l}\text { Licenciados (1), diplomados (4), } \\
\text { bachiller y FP (2), graduado escolar (1). }\end{array}$ & Aumento & Necesidad de mayor estabilidad laboral y de infraestructura \\
\hline Extremadura & Insuficiente. & $\begin{array}{l}\text { Licenciados (3 psicólogos y } 1 \text { pedagogo) } \\
\text { y } 24 \text { técnicos municipales. }\end{array}$ & Aumento. & Falta de implicación de los educadores y los ayuntamientos. \\
\hline Galicia & Insuficiente. & $\begin{array}{l}\text { Licenciados (53), diplomados (29), } \\
\text { bachiller y FP (10). }\end{array}$ & Aumento. & $\begin{array}{l}\text { Continuidad, estabilidad, dependencia de distintas instituciones que no conceden } \\
\text { la misma prioridad a esta temática y necesidad de colaboración entre instituciones. }\end{array}$ \\
\hline Melilla & & Técnicos. & & \\
\hline Murcia & Insuficiente. & $\begin{array}{l}\text { Licenciados (4), diplomados (3), bachiller } \\
\text { y FP (1) }\end{array}$ & Aumento. & \\
\hline Navarra (1) & Insuficiente. & & Aumento. & $\begin{array}{l}\text { Integración de profesionales en las estructuras municipales, de los programas } \\
\text { de prevención en los Servicios Sociales de forma normalizada, de la educación } \\
\text { para la salud de forma transversal en el ámbito educativo y falta de formación. }\end{array}$ \\
\hline La Rioja & Insuficiente. & Licenciados, diplomados y graduado escolar. & Aumento. & Falta personal e insuficiente formación. \\
\hline Valencia & Suficiente. & $\begin{array}{l}\text { Licenciados (46), diplomados (14), } \\
\text { bachiller y FP (4). }\end{array}$ & Aumento. & Necesidad de un mayor nivel de formación. \\
\hline
\end{tabular}

(1) En líneas generales, es imposible determinar el perfil mayoritario, no obstante, en la Dirección Técnica del Plan Foral hay un médico para tareas de prevención; en el medio comunitario, todos los programas cuentan con educadores, tres con un psicólogo y todos los servicios de base tienen trabajadores sociales; por último, en el ámbito escolar, la figura de referencia se procura que sea el orientador del centro.

Fuente: elaboración propia a partir de los datos suministrados en el cuestionario de recogida de información a los responsables autonómicos. 


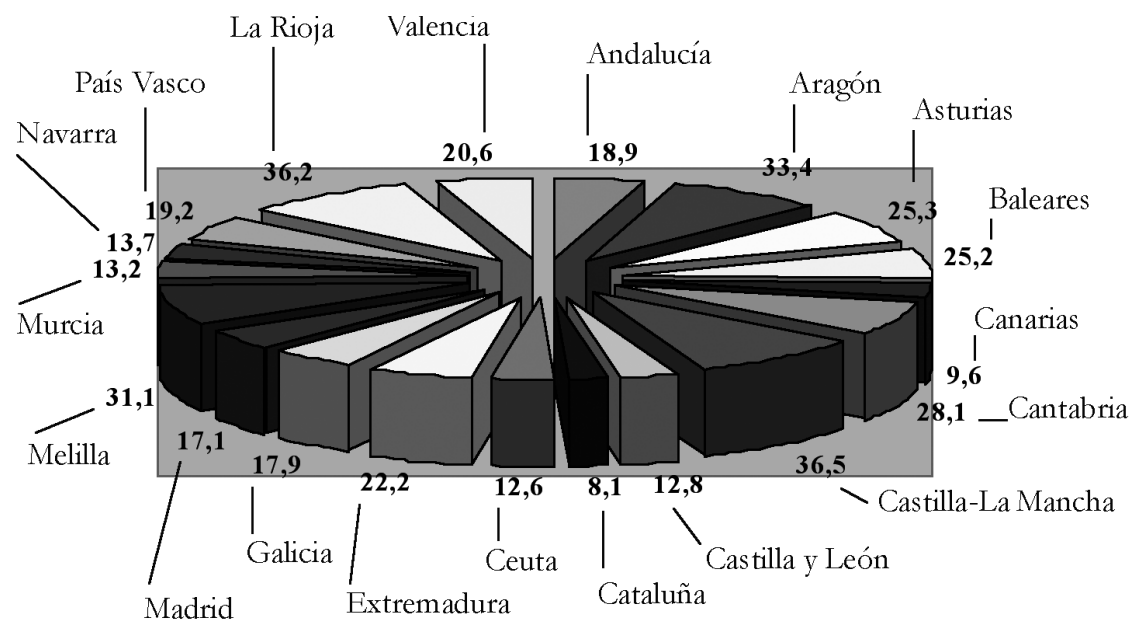

Gráfico II. Porcentaje del presupuesto total destinado al área de prevención, por comunidades (2003).

Asturias: datos de aquellas instituciones y entidades que han informado sobre el mismo.

Cataluña: no se incluyen gastos por programas con objetivos más amplios, aunque los drogodependientes también se beneficien de ellos.

Galicia: presupuesto correspondiente a la prevención y a la incorporación social, proveniente de los Fondos de la Comunidad Autónoma (FCA) y de los Presupuestos Generales del Estado (PGE).

Murcia: no se incluyen presupuestos municipales ni de otros organismos de la comunidad autónoma o de la Administración central.

Navarra: no se incluyen los gastos que financian los ayuntamientos.

País Vasco: cantidades dedicadas a intervenciones de carácter únicamente específico.

Fuente: elaboración propia a partir de la Memoria del PNSD, 2003.

ciones, reuniones institucionales, proyectos, acuerdos, grupos de trabajo y protocolos de colaboración.

Situación contraria se aprecia en la relación con las ONGs, donde es una pauta generalizada el desarrollo de convenios, conciertos o subvenciones. Estas organizaciones han ido adaptándose a las nuevas necesidades y especializándose hasta desarrollar, en estos momentos, tareas en las diferentes áreas de actuación. Se ha llegado a hablar del surgimiento de un movimiento asociativo con cierto carácter preventivo, que presta su colaboración en gran parte de los programas y actividades. En las comunidades, aunque es mayor su dedicación a tareas asistenciales, resalta el caso particular de Galicia, el País Vasco y Navarra, donde se le otorga una especial importancia a su labor en prevención.

\subsection{Resultados de las políticas preventivas: fortalezas, debilidades y retos}

Como última parte del análisis de los datos obtenidos de las encuestas y las entrevistas, se obtienen las principales fortalezas y debilidades de la prevención. En lo que respecta al primer caso, como fortalezas o aspectos positivos 
se han considerado todas aquellas características, circunstancias o acciones que favorecen un adecuado desarrollo de las intervenciones y su inserción en la sociedad, contribuyendo a reducir las probabilidades de que surjan problemas relacionados con el consumo de drogas. Al hablar de debilidades o aspectos negativos, nos referiremos, por el contrario, a aquellas situaciones que dificultan o hacen más probable el fracaso de la actuación.

Comenzando por las fortalezas (ver tabla V), sobresalen principalmente, en Andalucía y Baleares, los buenos resultados y el alcance de mayor población en los programas de prevención escolar, familiar y comunitaria. Esto es algo clave, es decir, aunque se trate de los campos con los que más frecuentemente se trabaja, su labor en la formación y preparación del individuo desde sus primeros años de vida para enfrentarse al complejo mundo de la droga es la más importante. En Aragón, despunta el modelo particular de «intervención preventiva» desarrollado, ya que hablamos de un patrón integral en el que se confunde asistencia y prevención. Esto fomenta un amplio abanico de posiciones técnicas y políticas basadas en el consenso (mejor comprensión del fenómeno de las drogas y de sus consecuencias sanitarias y sociales). Vemos, como diría Becoña (2002), parte de «una alianza con los distintos sectores implicados».

En Asturias, cabe resaltar el notable papel de los planes municipales sobre drogas y la excelente coordinación entre Salud y Educación, aspecto que no siempre resulta fácil en todo el territorio. En Canarias, Extremadura, Madrid y Murcia, es importante el creciente protagonismo de los ayuntamientos, lo que permite un mayor acercamiento a los ciudadanos y constituye un objetivo prioritario en la actualidad. En Cantabria, el sistema de acreditación de la calidad (AENOR) en todos sus servicios y áreas. Esta situación favorece el consiguiente reconocimiento público de la buena gestión y calidad en sus actuaciones. Según datos del Plan Regional, se ha mantenido la certificación obtenida desde el año 1999, renovando anualmente la acreditación, una política de compromiso con la mejora continua que ha de generalizarse. Por su parte, en Castilla-La Mancha, el elevado presupuesto destinado a esta área de actuación (un 36,5\% del total), lo que se encuentra en concordancia con la prioridad que se le otorga actualmente.

En Castilla y León, la implicación y coordinación con los planes locales sobre drogas y la penetración, continuidad y consolidación de los programas de prevención escolar. En Cataluña y el País Vasco, la sólida planificación, coordinación y evaluación. Tareas todas ellas no fácilmente ejecutables en el conjunto de los planes autonómicos. En Ceuta, la labor del Instituto Ceutí para la Prevención de las Drogodependencias (planificación), la coordinación y la cooperación, y, en Galicia, la gran cobertura del programa de enseñanza no universitaria y el papel de las ONGs. Estas últimas entidades han ido desarrollando labores cada vez más consolidadas y mejor planificadas en materia de prevención en el territorio. En el caso de Galicia, comunidad que nos ocupa en este momento, además de tareas de información y formación, se encargan de impulsar y participar en las estructuras y en los órganos de coordinación de programas y actividades y en el modelo de intervención y evaluación. Según 
Tabla V. Fortalezas, debilidades y retos de la prevención, por comunidades autónomas

\begin{tabular}{|c|c|c|c|}
\hline Comunidades & Fortalezas & Debilidades & Retos \\
\hline Andalucía & Prevención escolar, familiar y comunitaria. & Prevención laboral. & $\begin{array}{l}\text { Programas más potentes e interrelacionados. } \\
\text { Colectivos de riesgo. }\end{array}$ \\
\hline Aragón & $\begin{array}{l}\text { Modelo de «intervención preventiva» } \\
\text { (confunde asistencia y prevención). }\end{array}$ & $\begin{array}{l}\text { Evaluación de los programas } \\
\text { de prevención escolar y seguimiento } \\
\text { de los centros que los aplican. }\end{array}$ & $\begin{array}{l}\text { Incremento de la percepción de riesgo. } \\
\text { Herramientas trabajar el cánnabis. } \\
\text { Sectores cercanos a los jóvenes. }\end{array}$ \\
\hline Asturias & $\begin{array}{l}\text { Papel PMD (1). } \\
\text { Coordinación entre Salud y Educación. }\end{array}$ & La coordinación. & $\begin{array}{l}\text { Trabajo con S. Atención Primaria. } \\
\text { Aumento del presupuesto. } \\
\text { Familias de drogodependientes. }\end{array}$ \\
\hline Baleares & Prevención escolar, familiar y comunitaria. & El presupuesto. & Normalización de la prevención escolar en el marco Educación para la Salud. \\
\hline Canarias & Protagonismo de los ayuntamientos. & Tasas de admisiones a tratamiento. & Concienciación de la población. \\
\hline Cantabria & $\begin{array}{l}\text { Sistema de acreditación calidad AENOR } \\
\text { en todos servicios. }\end{array}$ & $\begin{array}{l}\text { La planificación. } \\
\text { Los recursos. } \\
\text { La prevención laboral. }\end{array}$ & $\begin{array}{l}\text { Jóvenes (13-16 años). } \\
\text { Generalización de la prevención escolar. } \\
\text { Evaluación del proceso y resultados. }\end{array}$ \\
\hline Castilla-La Mancha & Elevado presupuesto. & $\begin{array}{l}\text { Ámbitos comunitario } \\
\text { y de la comunicación. }\end{array}$ & $\begin{array}{l}\text { Programas comunitarios. } \\
\text { Colectivos de riesgo. } \\
\text { Coordinación de la atención primaria. }\end{array}$ \\
\hline Castilla y León & $\begin{array}{l}\text { PLD (2). } \\
\text { Prevención escolar. }\end{array}$ & $\begin{array}{l}\text { RRHH. } \\
\text { Prevención familiar. }\end{array}$ & $\begin{array}{l}\text { RRHH (aumento). } \\
\text { Prevención familiar y laboral. } \\
\text { Programas más rigurosos. }\end{array}$ \\
\hline Cataluña & Planificación, coordinación y evaluación. & Recursos (humanos y económicos). & $\begin{array}{l}\text { Evaluación de los programas escolares. } \\
\text { Implicación de los colectivos. }\end{array}$ \\
\hline Ceuta & $\begin{array}{l}\text { Instituto Ceuti para la Prevención. } \\
\text { Coordinación y cooperación. }\end{array}$ & $\begin{array}{l}\text { Identificación de poblaciones de riesgo. } \\
\text { Desarrollo de diferentes ámbitos. }\end{array}$ & Estabilidad laboral del personal. \\
\hline Extremadura & Protagonismo de los ayuntamientos. & $\begin{array}{l}\text { Evaluación de los programas } \\
\text { de prevención escolar y seguimiento } \\
\text { de los centros que los aplican. }\end{array}$ & $\begin{array}{l}\text { Creación de comisiones. } \\
\text { Contratación de técnicos municipales. }\end{array}$ \\
\hline
\end{tabular}


Tabla V. Fortalezas, debilidades y retos de la prevención, por comunidades autónomas (continuación)

\begin{tabular}{|c|c|c|c|}
\hline Comunidades & Fortalezas & Debilidades & Retos \\
\hline Galicia & $\begin{array}{l}\text { Cobertura del Programa de Enseñanza } \\
\text { no Universitaria. } \\
\text { Papel de las ONG. }\end{array}$ & $\begin{array}{l}\text { RRHH. } \\
\text { Prevención laboral. }\end{array}$ & $\begin{array}{l}\text { Formación de los profesionales. } \\
\text { Educación en valores et. Riesgo. } \\
\text { Aumento de los programas de prevención escolar. }\end{array}$ \\
\hline Madrid & Protagonismo de los ayuntamientos. & Presupuesto. & Presupuesto. \\
\hline Melilla & $\begin{array}{l}\text { Consolidación de los programas. } \\
\text { Presupuesto. }\end{array}$ & RRHH. & Prevención en todos sus ámbitos (elevado nivel de consumo). \\
\hline Murcia & Protagonismo de los ayuntamientos. & RRHH. & Estudios sobre evaluación progresiva. \\
\hline Navarra & Integración y normalización prevención. & $\begin{array}{l}\text { La coordinación. } \\
\text { Prevención laboral. }\end{array}$ & $\begin{array}{l}\text { Prevención familiar (cambio de actitudes). } \\
\text { Normalización de las drogas. }\end{array}$ \\
\hline País Vasco & Planificación, coordinación y evaluación. & Prevención laboral. & $\begin{array}{l}\text { PLD (2). } \\
\text { Inv. evaluación resultados. } \\
\text { Información. }\end{array}$ \\
\hline La Rioja & $\begin{array}{l}\text { Oficina de Atención al Ciudadano. } \\
\text { Aumento de las subvenciones locales. }\end{array}$ & Prevención escolar, laboral y familiar. & $\begin{array}{l}\text { Aumento subvenciones. } \\
\text { Línea: ocio y tiempo libre. } \\
\text { Colectivos de riesgo. } \\
\text { Prevención Laboral y Comunicación. }\end{array}$ \\
\hline Valencia & $\begin{array}{l}\text { Red fuertemente financiada. } \\
\text { Prev. escolar y comunitaria. }\end{array}$ & $\begin{array}{l}\text { Prevención escolar (inclusión } \\
\text { de los programas en la escuela). }\end{array}$ & $\begin{array}{l}\text { UPC en municipios pequeños. } \\
\text { Debates más científicos. }\end{array}$ \\
\hline
\end{tabular}

(1) Planes municipales sobre drogas.

(2) Planes locales sobre drogas.

Fuente: elaboración propia. 
datos de su plan, algunos servicios son gestionados por varias ONGs y, en el año 2002, se habían realizado convenios o conciertos con veintinueve organizaciones de esta índole.

Para finalizar, en Melilla, la consolidación de los programas y el elevado presupuesto (un 31,1\% del total); en Navarra, la integración y normalización de la prevención (objetivo clave en estos momentos); en La Rioja, la Oficina de Atención al Ciudadano y el aumento de las subvenciones a corporaciones locales y, en Valencia, la existencia de una red fuertemente financiada y la extensión de la prevención escolar y comunitaria. Como se enuncia en el Plan Autonómico Valenciano (1999-2002), en los últimos años se ha producido un notable incremento de centros, programas y servicios (consolidación del Plan de Prevención Escolar: 1.181 centros de Educación Secundaria en el 2002 e implantación definitiva de la red de Unidades de Prevención Comunitaria, que dota de cobertura a todos los municipios de más de 20.000 habitantes).

Como contrapartida, entre los déficits más relevantes (ver tabla V), en Andalucía y el País Vasco, se distingue el escaso desarrollo de la prevención laboral. En general, al analizar los programas y las actividades de este ámbito, se aprecia una menor expansión respecto al resto. Pese al mayor trabajo y dedicación con el paso del tiempo, todavía no se cuenta con claras líneas de actuación enfocadas al mismo. Así, se hace necesaria la articulación de medidas que permitan la integración en los lugares de trabajo del enfermo para conseguir el fin último del proceso de inserción social. En Aragón y Extremadura, la no-evaluación de los programas de prevención escolar ni el seguimiento de los centros que los aplican. Según datos de la Memoria del PNSD (2002: 20), de los 69 programas realizados en las diferentes comunidades, 53 han elaborado algún tipo de evaluación (el 76\% de los mismos), de lo que se deduce el papel básico que ha de ocupar esta práctica. En Asturias, la falta de coordinación; en Baleares y Madrid, el presupuesto (como veíamos en el análisis, un 25,2\% del total, en el caso de la primera, y un 17,1\% en el de la segunda), y, en Canarias, las elevadas tasas de admisiones a tratamiento, fundamentalmente por opiáceos y cocaína $^{13}$. Las actuaciones hacia los consumos recreativos constituyen un campo ciertamente nuevo y poco desarrollado en el momento presente.

En Cantabria, la falta de planificación (especialmente en el trabajo con los sectores de riesgo), de recursos y la prevención laboral. En este último caso, se precisa que todas las actuaciones se encuentren encuadradas en los planes de seguridad e higiene en las empresas, el desarrollo de adecuados cauces de coordinación entre patronal y sindicatos y las intervenciones con los colectivos de riesgo. En Castilla-La Mancha, la escasa implantación de los ámbitos comunitarios y de la comunicación ${ }^{14}$. A su vez, en Castilla y León, carencias en los recursos humanos (en cuanto al número, a la formación y a la especialización)

13. Datos que coinciden con los reflejados por la Encuesta Domiciliaria sobre el Consumo de Drogas (PNSD, 2003).

14. Según los responsables del plan, no se determinan los mensajes preventivos que se van a transmitir. 
y en la prevención familiar. En Cataluña, la falta de personal y de presupuesto (un $8,1 \%$ del total), y en Ceuta, la dificultad de identificación de poblaciones de intervención prioritaria o de riesgo y el desarrollo de los diferentes ámbitos de intervención. Actualmente, al objeto de sensibilizar a la sociedad sobre esta problemática, se ha de promover un consenso basado en el compromiso de todas y cada una de las áreas implicadas en la prevención.

En Melilla, Murcia y Galicia, la escasez de recursos humanos y, en ésta última, además, la prevención laboral (no lo suficientemente enfocada a los colectivos de alto riesgo). En La Rioja, la falta de integración de la prevención en los currículos escolares, en la empresa y en la familia; en Navarra, la coordinación y el ámbito laboral (no se aprecia una línea de trabajo establecida desde el plan para promoverla), y, en Valencia, la dificultad de incluir los programas en la escuela. La prevención escolar continúa siendo el ámbito de intervención preferente en el territorio nacional, y la generalización de sus programas a toda la población estudiantil se defiende como una de las estrategias más importantes para enfrentarse al problema de las drogas.

En último lugar, haciendo una valoración global de la información y los datos expuestos hasta el momento, los retos estarían encaminados (ver tabla $\mathrm{V}$ ), en Andalucía, a construir programas más potentes e interrelacionados y al trabajo con los colectivos de riesgo (punto que coincide con uno de los objetivos prioritarios en el actual II Plan Andaluz sobre Drogas y Adicciones 20022007). En Asturias, a favorecer el trabajo con los servicios de atención primaria, es decir, a la potenciación de los servicios sociales generales o comunitarios, por su importancia en la prevención y la atención a los problemas derivados del consumo; a aumentar el apoyo económico (reto también presente en Madrid) y al compromiso con las familias de drogodependientes. En este último caso, se trata de incidir en el binomio formado por familia y droga cuando ya está presente el problema y, por tanto, en las posibles consecuencias asociadas (económicas, falta de comunicación, violencia, desestructuración del hogar, etc.) (véase, por ejemplo, Grover, 1998). En Castilla-La Mancha, a generalizar los programas comunitarios, enfatizando el trabajo con los colectivos de riesgo y la coordinación con los equipos de atención primaria. En Castilla y León, a aumentar los profesionales, especialmente los dedicados a las tareas de supervisión, apoyo y seguimiento técnico de los mediadores formados para la prevención; a impulsar una línea rigurosa, sistemática y evaluada de prevención familiar; a formalizar y universalizar las actuaciones en el ámbito laboral, y, en definitiva, a estrechar este campo y conseguir una prevención basada en la evidencia y en la ciencia, desarrollando programas completamente evaluados (desafío también compartido con Cantabria, Murcia y el País Vasco).

Enlazando con este último aspecto, generalizable al conjunto del territorio, según Amador Calafat (1993), en España existen instrumentos y programas completamente evaluados y que, sin embargo, no son aplicados, sino que se llevan a la práctica otros que no cumplen los criterios exigibles en esta materia. Esto lleva a la necesidad de realizar revisiones técnicas y de estudiar el coste que supondría desarrollar programas no evaluados, entre ellos, no conocer sus 
efectos futuros, en un tema tan importante y de tan graves consecuencias en la actualidad. Por estos motivos, hay que favorecer el desarrollo de programas con una metodología rigurosa y susceptible de evaluación, más aún si cabe que se dispone de ellos.

Continuando con los retos, en Cataluña, se estudia la evaluación de las intervenciones escolares y la formación e implicación de los profesionales, las entidades y las asociaciones de jóvenes. Una de las líneas de trabajo más novedosas - desarrollada fundamentalmente por municipios, planes autonómicos y varias ONG - se centra en la juventud (colectivo diana en Aragón, Canarias, Cantabria y Melilla) y en la promoción de actividades de ocio alternativo durante los fines de semana. En Ceuta, la estabilidad laboral del personal y, en Extremadura, la creación de comisiones y la contratación de técnicos municipales.

En Galicia, se persigue la formación continua de los profesionales, el reforzamiento de la educación en valores en las etapas de riesgo y el aumento del número de programas de prevención escolar (también presente en Baleares ${ }^{15} \mathrm{y}$ Cantabria). En el contexto europeo, todos los estados miembros otorgan una importante prioridad a la prevención, aunque el mayor hincapié se realiza en las escuelas (promoción integral de la salud, desarrollo de aptitudes sociales y personales, etc.) (FAD, 1997). Se trata de un ámbito idóneo en la detección temprana de posibles factores de riesgo y un básico agente de socialización (véase Alonso, Freijo y Freijo, 1996). En Navarra, se pretende modificar la actitud de los padres y normalizar las drogas, esto es convivir con ellas, cambiando el enfoque de los técnicos que vienen de programas libres de drogas donde la idea es "no consumir». Se ha producido una generalización en un corto periodo de tiempo, una «normalización» de estas sustancias, una integración de las mismas en la vida cotidiana de la sociedad, un amplio grupo de consumidores adaptados socialmente (Calafat y otros, 2000).

En La Rioja, se quiere aumentar las subvenciones y financiar las escuelas de padres, hacer llegar materiales a los centros, fomentar los espacios de ocio y tiempo libre ${ }^{16}$ (programa de alcohol en jóvenes), incidir en los grupos de riesgo y en los menores, promover la prevención laboral y desarrollar toda la información a través de Internet, en el portal de drogas. El papel de los medios de comunicación es cada vez más relevante, ya que gran parte del conocimiento de la realidad se realiza a través de los mismos. Coincidiendo con SánchezMartos (1997), suponen una aportación esencial en la consecución de los objetivos de las campañas de prevención sobre uso indebido de drogas. La principal clave estriba en la gran capacidad que tienen de aumentar el conocimiento de los individuos sobre los diferentes temas, proporcionándoles información y sensibilizándoles sobre hábitos y prácticas, tanto nocivas o de riesgo, como positivas o saludables. En último lugar, en Valencia, se pretende disponer de

15. Según datos del PNSD, desde el año 2000 se persigue la implantación de los programas de prevención escolar de manera normalizada en el marco de la educación para la salud.

16. Para un estudio más pormenorizado de los jóvenes y el ocio, véase Comas (coord.), 2003, o Acero et al. (2002). 
una unidad de prevención comunitaria (UPC) en municipios cada vez más pequeños y lograr debates más científicos y profesionales. Esta última idea ha de descansar sobre la construcción de un modelo teórico, el conocimiento de los factores de riesgo y la evaluación de los programas preventivos (Becoña, 2002).

\section{Aportaciones finales. Conclusiones}

La prevención del consumo de drogas ha pasado a ser el objetivo prioritario para la mayor parte de las autonomías. Cuenta con marcos de actuación integrados y cada vez más consensuados entre todas las entidades implicadas en su ejecución. A su vez, se han conseguido importantes avances en su normalización e integración, tanto en el currículo escolar - llega a más edades y sectores-, como su enmarque en una estrategia más global de promoción de la salud y el bienestar, uno de los objetivos prioritarios en el presente. Junto con ello, se ha dado énfasis al desarrollo y al aumento de los programas, actividades, recursos y destinatarios, y la creciente participación de los municipios y del movimiento asociativo, que es cada vez más necesaria. Se aprecia una notable evolución de la prevención desde sus inicios hasta la actualidad, momento en el que contamos con intervenciones basadas en la evidencia y con programas cada vez más estructurados y científicamente contrastados. Según Burkhart (2004), responsable del área de prevención del Observatorio Europeo de la Droga y las Toxicomanías (OEDT), la evaluación en Europa es escasa y muestra determinadas carencias, fundamentalmente en materia de recursos. Sin embargo, en este contexto, destacan positivamente España e Inglaterra.

Junto a los avances conseguidos, todavía hay aspectos sobre los que habría que seguir incidiendo en años venideros. Partiendo de la base de que el principal inconveniente es que se está hablando de un área especialmente complicada al verse los resultados a muy largo plazo, sobresale, coincidiendo con Becoña (1999), la dificultad de sensibilizar a la sociedad y de llegar a todos los ciudadanos, especialmente a los jóvenes y a las familias (ver Velleman, Mistral y Sanderling, 2000 $)^{17}$, y algunas controversias en la planificación. En este último sentido, se registran problemas de coordinación (entre departamentos, profesionales y equipos de atención primaria), aspecto que debería ser revisado de cara al adecuado desarrollo de las acciones preventivas. Además, también se encuentran dificultades relacionadas con los recursos humanos (insuficiencia de personal y falta de formación) y económicos, y de evaluación, fundamentalmente de los programas de prevención familiar. Este último aspecto sobresale como uno de los campos con especiales dificultades, siguiendo a

17. Pueden consultarse algunas propuestas de acción, entre ellas, una información básica y sesiones de concienciación para la población general; amplios cursos centrados en la habilidad de los padres y en la vida con los adolescentes, y otros más intensivos para las familias de alto riesgo, etc. 
Acero (1997), se observa un déficit en la planificación de las acciones, en el marco conceptual de referencia y en la práctica inexistencia de afán evaluativo en estas iniciativas.

Por último, se perciben carencias en la definición e identificación de los sectores de intervención prioritaria (elaboración de mapas de riesgo). Se trata de conocer los colectivos de los que debería partir cualquier actuación. Según sugieren Hawkins, Catalano y Millar (1992), el camino más prometedor para encontrar estrategias efectivas para la prevención es a través de la investigación enfocada en el riesgo. Por ello, resulta necesario identificar estos factores en el abuso de drogas, conocer los métodos a través de los cuales han sido efectivamente tratados y la aplicación de los mismos a apropiadas muestras de población general y de alto riesgo en estudios controlados.

Por todos estos motivos, todavía hay que seguir trabajando. Puede ser relevante el hecho de entender la prevención como una tarea del conjunto de la sociedad que implica a todos los sectores, especialmente a los adolescentes y jóvenes - lo cual incrementa su percepción del riesgo-y a las familias — principales agentes preventivos (véase, entre otros, Comas, 1997; Ríos, Espina y Baratos, 1997; Orte, 1995)—. A continuación, es importante que la prevención se incorpore de manera normalizada y estable, de manera que llegue a todas las edades; que aumenten y se especialicen los recursos humanos, pilar clave en la actual política social, y que se incida en la labor con los padres, las asociaciones y los municipios con planes de drogas, por su papel privilegiado de cercanía al individuo. Como primer foco de actuación, se precisa enfatizar el papel del ámbito local (Vega, 2000). Junto con ello, es necesario promover la investigación en prevención, impulsando una línea rigurosa, sistemática y evaluada de todos sus programas, una realidad imperante y cada vez más requerida en el ámbito internacional.

\section{Referencias bibliográficas}

ACERO, A. (1997). «Familia y factores de protección: una estrategia preventiva». Revista de Estudios de Juventud, 40, 61-68.

Acero, A.; Moreno, G.; Moreno, J.; SÁnCheZ, L. (2002). La prevención de las drogodependencias en el tiempo de ocio. Manual de formación. Madrid: Asociación Deporte y Vida.

Alonso, D.; Freijo, A.; FreIjo, E. (1996). Actuar es posible: La prevención de las drogodependencias en la comunidad escolar. Madrid: Ministerio del Interior. Delegación del Gobierno para el Plan Nacional sobre Drogas.

Alvira, F. (2000). Manual para la elaboración y evaluación de programas de prevención del abuso de drogas. Madrid: Agencia Antidroga.

Arana, X.; MARKeZ, I.; Vega, A. (coords.) (2000). Drogas: cambios sociales y legales ante el tercer milenio. Madrid: Dykinson, 165-187.

Arenilla, M. (2000). «Planificación y políticas públicas». En: TRINIDAD, A. (coord.). Evaluación y calidad en las organizaciones públicas. Madrid: INAP.

BECOÑA, E. (2002). Bases científicas de la prevención de las drogodependencias. Madrid: Ministerio del Interior. Delegación del Gobierno para el PNSD. 
- (1999). Bases teóricas que sustentan los programas de prevención de drogas. Madrid: Ministerio del Interior. Delegación del Gobierno para el PNSD.

BURKHART, G. (2004). "Las políticas sobre drogas en el marco de la Unión Europea». Seminario: Las Políticas Públicas sobre Drogas: Análisis y Evaluación. Granada: Facultad de CCPP y Sociología. Enero.

- (1998). "Para qué evaluar». Observatorio Vasco de Drogodependencias. Centro de Documentación de Drogodependencias (CDD), Boletín, 44.

CALAFAT, A. (1993). "Evaluación del programa de prevención "Tú decides"». Adicciones, vol. 1(2).

Calafat, A.; Juan, M.; Becoña, E.; Fernández, C.; Gil, E.; Palmer, A. y otros (2000). Salir de marcha y consumo de drogas. Madrid: Ministerio del Interior. Plan Nacional sobre Drogas.

Center for Substance Abuse Prevention (CSAP) (1998). Preventing Substance Abuse Among Children And Adolescents: Family-Centered Approaches Prevention Enhancement Protocols System (PEPS). Rockville: DHHS Publication.

Comas, D. (coord.); Aguinaga, J.; Orizo, F.; Espinosa, A.; Ochaita, E. (2003). Jóvenes y estilos de vida. Valores y riesgos en los jóvenes urbanos. Madrid: Fundación de Ayuda contra la Drogadicción (FAD) e Instituto de la Juventud (INJUVE).

- (1997). "Sociología de las drogas en la sociedad española». En: SiRvENT, C.; RodrígueZ, J. (coords.). Drogodependencias. Madrid: Instituto Spiral, p. 39-74.

Delegación del Gobierno para el Plan Nacional sobre Drogas (2003). Encuesta domiciliaria sobre el consumo de drogas. Madrid: Ministerio de Sanidad y Consumo.

- (1996, 1997, 1998, 1999, 2001, 2002 y 2003). Memorias. Madrid: Ministerio del Interior.

- (1999). Estrategia Nacional sobre Drogas (2000-2008). Madrid: Ministerio del Interior.

Fundación de AyUda contra la Drogadicción (FAD) (1997). Progresos en el análisis de datos para la investigación de intervenciones reventivas. Madrid.

- (1997). 2002. Informe anual para la prevencion de drogodependencias. Madrid, p. 89.

Grover, P. L. (ed.) (1998). Preventing abuse among children and adolescents: Family-centered approaches. Referente guide. Washington: DHHS Publication No. (SMA) 3223-FY98, p. 1.19-1.27.

Hawkins, J. D.; Catalano, R. F.; Miller, J. Y. (1992). «Risk and Protective Factors for Alcohol and Other Drug Problems in adolescence and Early Adulthood: Implications for Substance Abuse Prevention». Psychological Bulletin, 112 (1).

Kelle, U. (2001). «Explicaciones sociológicas entre micro y macro y la integración de métodos cualitativos y cuantitativos». Forum Qualitative Research, vol. 2, no 1. <www.qualitative-research.net/fqs-texte/1-01/1-0kelle-s.htm>.

KRÖGER, CH.; WinTER, H.; SHAW, R. (1998). Guía para la evaluación de las intervenciones preventivas en el ámbito de las drogodependencias: Un manual para responsables de planificación y evaluación de programas. Munich: Instituto para la Investigación de la Terapia (IFT).

Leukefeld, C. G.; BuKOSKI, W. J. (1997). Métodos de investigación evaluativa de programas preventivos: hallazgos y consensos. Monografías de Investigación: Estudios sobre intervenciones en prevención del abuso de drogas: aspectos metodológicos. Madrid: Plan Nacional sobre Drogas, p. 255-261.

MejÍAS, E. (coord.) (2000). La percepción social de los problemas de drogas en España. Madrid: Fundación de Ayuda contra la Drogadicción (FAD). 
- (dir.); Comas, D.; Elzo, J.; Megías, I.; Navarro, J.; Rodríguez, E.; Romani, O. (2000). Los valores de la sociedad española y su relación con las drogas. Barcelona: Fundación La Caixa. Colección Estudios Sociales; 2.

Observatorio Vasco sobre Drogas (2001). Informe 4. Viceconsejería de Asuntos Sociales.

ORTE, C. (1994). Familia y drogodependencias: Una guia documental. Palma de Mallorca: Universidad de Baleares, p. 12-27.

RECIO, J. L. (1997). «El fenómeno de la droga como capital simbólico y hecho social total». Revista de Estudios de Juventud, 40: 9-16.

Ríos, J. A.; Espina, A.; Baratas, M. D. (1997). Actuar es posible: la prevención de las drogodependencias en la familia. Madrid: Ministerio del Interior. Delegación del Gobierno para el Plan Nacional sobre Drogas.

SAlVADOR, T. (2001). «Evaluación en el ámbito preventivo: avances y retos de futuro». Adicciones, 13 (Supl. 1): 50.

SÁNCHEZ-MARTOS, J. (1997). «Los medios de comunicación social ante el fenómeno de la drogodependencia». Revista de Estudios de Juventud, 40: 69-78.

THE SIGNIFICANCE OF DEFINITIONS OF SUCESS IN PROGRAM EVALUATION (2000). The Evaluation Review, 24, no 5.

Trinidad, A. (dir.); Alemán, C.; Castón, P. (cols.); Fernández Alonso, M.; MartínLAGOS, M. D. (2003). Evaluación y diagnóstico de los planes autonómicos sobre drogas. Madrid: Ministerio del Interior, PNSD.

VAn DER STel, J. (ed.); VoORdeWIND, D. (ass.) (1998). Handbook Prevention; Alcohol, Drugs and Tobaco. Pompidou Group-Council of Europe \& Jellinek Consultancy. Velleman, R.; Mistral, W.; SANDERLING, L. (2000). Taking the message home: involving parents in drugs prevention. Londres: Drugs Prevention Advisory Center, Home Office. <www.pnsd.msc.es>. 